\title{
Molecular mechanisms of glucocorticoids action: implications for treatment of rhinosinusitis and nasal polyposis
}

\author{
Alicja Grzanka • Maciej Misiołek • \\ Wojciech Golusiński · Jerzy Jarząb
}

Received: 1 April 2010/Accepted: 25 June 2010/Published online: 14 July 2010

(C) The Author(s) 2010. This article is published with open access at Springerlink.com

\begin{abstract}
Intra-nasal glucocorticoids are the most effective drugs available for rhinosinusitis and nasal polyposis treatment. Their effectiveness depends on many factors and not all of them have been well recognized so far. The authors present the basic information on molecular mechanisms of glucocorticoid action, direct and indirect effects of glucocorticoids on transcription of genes encoding inflammatory mediators. They focus on recently proved nongenomic mechanisms which appear quickly, from several seconds to minutes after glucocorticoid administration and discuss clinical implications resulting from this knowledge. Discovery of nongenomic glucocorticoid actions allows for better use of these drugs in clinical practice.
\end{abstract}

Keywords Glucocorticoids .

Nongenomic and genomic action · Nasal polyps ·

Rhinitis

A. Grzanka $(\bowtie) \cdot$ J. Jarząb

Clinical Department of Internal Diseases,

Dermatology and Allergology, Zabrze,

Medical University of Silesia, M.C.Skłodowskiej 10,

41-800 Katowice, Poland

e-mail: alicjag@mp.pl

M. Misiołek

Clinical Department of Laryngology, Zabrze,

Medical University of Silesia, Katowice, Poland

W. Golusiński

Department of Head and Neck Surgery and Laryngological

Oncology, The Greater Poland Cancer Center,

Poznan University of Medical Sciences, Poznan, Poland

e-mail:wgolusinski@ump.edu.pl

\section{Introduction}

Rhinosinusitis and nasal polyposis are inflammatory diseases of nasal and sinuses mucosa leading to limited nose patency, excessive secretion, anosmia, headaches, and considerable deterioration of quality of life [1-4]. They coexist or become risk factors for development of other diseases, including asthma. Appropriate and early treatment can bring notable benefits to patients. In rhinosinusitis and nasal polyposis treatment, intra-nasal glucocorticoids (inGC) are the most effective drugs available. Intra-nasal glucocorticoids are recommended not only for allergic rhinitis (AR), but can be useful in the treatment of some forms of nonallergic rhinitis (NR) [5]. These comprise the first-line treatment for rhinosinusitis [3]. The knowledge of mechanisms of glucocorticoids (GC) action helps in their application in appropriate clinical situations as using GC is not effective to the same extent in all patients. However, even though we have been using glucocorticoids for over 60 years, their exact mechanism of action is still under discussion. Recently, GC nongenomic actions are considered which can play an important role for the inhaled (intranasal and intrabronchial) treatment. This fact was emphasized by the document "Allergic Rhinitis and its Impact on Asthma (ARIA)" listing nongenomic effects as one of the three desirable pharmacological properties of inGC [6]. However, the clinical significance of nongenomic mechanisms of GC effects is still not well understood and the reports considering upper airways diseases are scarce.

\section{Glucocorticoid receptor}

On the cell level each GC functions through the same receptor mechanism. The glucocorticoid receptor (GCR) 
molecule transfers information signal brought by a hormone or a drug (called ligand) mainly by changing gene expression regulation. GCR is present in all tissues except anuclear cells. Human airways are characterized by exceptionally high levels of GCR expression. Within the nose, the presence of GCR is found in all cells, by the greatest number in epithelial cells, in submucous glands and in leucocytes [7].

\section{Glucocorticoid receptor heterogenity}

The GCR gene is localized on the fifth chromosome and is composed of nine exons. As a result of alternative splicing of GCR primary transcript (mRNA), four variants, called isoforms, may be produced. The only active isoform is $\operatorname{GCR} \alpha$, while $\operatorname{GCR} \beta, \operatorname{GCR} \delta$, and $\operatorname{GCR} \gamma$ retain only some features of full-activity spectrum. GCR $\beta$ is shorter by 35 amino acids and differs by sequence of other 15 amino acids from the classic GCR $\alpha$ receptor. The differences relate to GC binding domain, therefore a total lack of ability to bind ligand has been reported for this receptor variant. But yet, it can bind DNA, thus it blocks competitively GCR $\alpha$ access to DNA, not being able to activate gene expression. Moreover, GCR $\beta$ can bind directly to the active $\operatorname{GCR} \alpha$ monomer creating nonactive heterodimer. This additionally inhibits GCR $\alpha$ activity. Additionally, the half-time period of the GCR $\beta$ variant is two times longer than that of GCR $\alpha$. Therefore, GCR $\beta$, holding an antagonist role, can inhibit signaling pathways of active GCR $\alpha$ and can cause a secondary resistance to GC. It was shown that proinflammatory cytokines (IL-1 IL-2, IL-4, IL-13 and TNF $\alpha$ ), whose participation in polyps and rhinitis pathogenesis is well substantiated, activate mechanisms of alternative splicing of mRNA [8-10]. Therefore, inflammatory processes can increase GCR $\beta$ expression. With the protracting inflammation, final $\operatorname{GCR} \beta$ density increases, therefore GC sensitivity can get decreased. In nasal polyps, which are not treated by GC, significantly higher GCR $\beta$ expression occurs in comparison with the unchanged mucosa [11]. Application of inGC and systemic GC for 2 weeks in those patients, not having any influence on $\operatorname{GCR} \beta$, significantly increases GCR $\alpha$ expression in comparison with the level shown before treatment and with the group which did not get GC. A beneficial consequence for a patient is an increase of GCR $\alpha$ to GCR $\beta$ ratio. This might be the way how the high GC doses are able to break the resistance/decreased sensitivity to $\mathrm{GC}$, which can be a mechanism of so called medical polypectomy [3]. This could be the reason why GC with their strong anti-inflammatory properties are so far the only group of drugs which can effectively cause a reduction in the size and symptoms of polyps and prevent their recurrence after surgeries [3, 4].
There is evidence of a dose-response in this effect [12]. Moreover, a delay in decision on GC treatment may cause failure of nasal polyposis treatment.

Biologic importance of GCR $\delta$ and GCR $\gamma$ is still being investigated. Studies suggest that there are many other more or less active forms of GCR and their expression depends on a type of stimulus and tissue [13]. Final explanation of post-transcriptional gene modifications (variants of alternative GCR splicing) as reasons for differences in GC sensitivity requires further study. However, it seems that the effectiveness of GC treatment is influenced by keeping appropriate proportions between isoforms rather than by their absolute level.

\section{Intracellular localization of glucocorticoid receptor}

The unusually wide range of GC actions can be explained by GCR presence in three cell compartments: nucleus, cytoplasm, and plasma membrane (Fig. 1).

Initially, it was thought that the primary localization of GCR was cytoplasm (cGCR) which moves to the nucleus only after binding the ligand. Now it is clear that the GCR stays in the nucleus (nGCR). nGCR occurs only in the form of dimer and after activation by the ligand interacts with DNA to intensify or inhibit gene transcription, directly. In cytoplasm GCR occurs as monomer, which enables the protein-protein interaction with other extranuclear regulatory proteins and, indirectly, gene transcription modification. Both types of action, cytoplasmatic and nuclear, are defined as genomic ones since they take place through regulation of gene activity. The possibility of GCR occurrence in cell membrane has been postulated for a long time, but its presence in this cell area was confirmed only in 2004 thanks to immunofluorescence staining with the

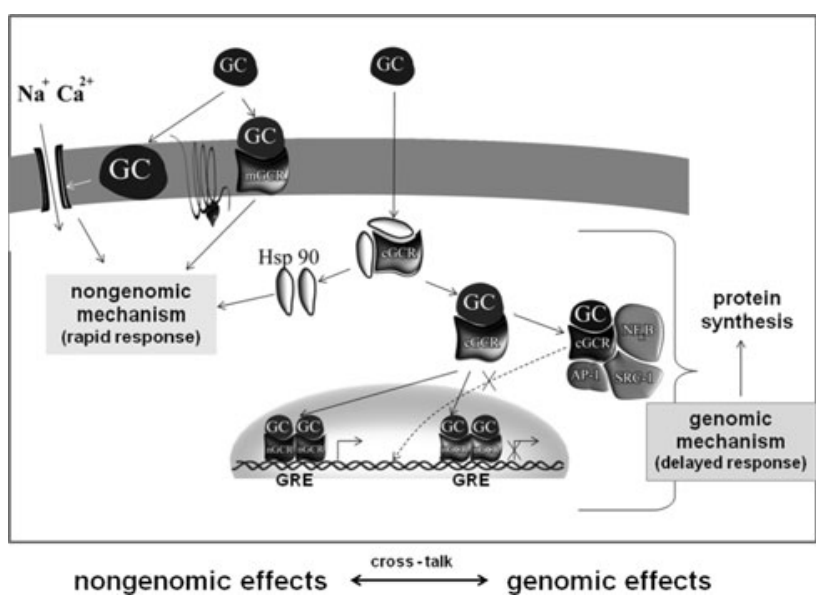

Fig. 1 Intracellular localization of glucocorticoid receptor (plasma membrane, cytoplasm and nucleus) and nongenomic and genomic mechanism of glucocorticoids actions 
use of liposomes [14]. Membrane GC receptors (mGCR) initiate rapid changes in activity of various signal pathways when activated by GC. These mechanisms are called nongenomic ones.

\section{Genomic mechanisms of glucocorticoid action}

The classical model of GC action includes their influence (direct or indirect) on gene transcription and translation, for many specific genes encoding inflammatory mediators (Fig. 1). Genomic effects appear not earlier than after tens of minutes. The ARIA directives for inGC define this time as 7-8 $\mathrm{h}$ following administration [6]. The time for reaching maximal effectiveness can be even up to 2 weeks [6].

In the cytoplasm, the nonactive receptor is present in a complex with chaperones. The GC-activated GCR undergoes an initial conformation change, resulting in dissociation of the chaperone-GCR complex. In this way the GC-GCR complex is activated and can translocate to the nucleus where it dimerizes. Only as a homodimer it can bind to the DNA regulatory sequences, called GC response elements (GRE) and found in the promoter regions of glucocorticoid-regulated genes. If the GC-GCR complex binding leads to gene activation, the GRE sequence is termed as "positive" GRE. GC causes activation of the genes listed in Table 1. Negative GRE is also described where GC-GCR leads to gene suppression (direct repression). So far, the presence of negative GRE in the promotor region was proved only for a few genes (Table 1).

The GC-GCR and DNA binding provides a signal for coactivator $\mathrm{CBP}$ proteins (CREB binding protein), $\mathrm{pCAF}$ (p300-CBP associated factor) and SRC-1 (steroid receptor coactivator-1). Coactivator proteins with inner histone acetyltransferase (HAT) activity loosen the DNA strand and facilitate RNA polymerase II and TATA-box binding protein (TBP) actions, which conditions initiation of transcription [15].

Many genes may be influenced by GC without direct interaction with DNA (Table 1). It happens through binding of factors important for inflammatory process development, often coactivators or corepressors of other genes' transcription. The process of gene transcription inhibition without DNA binding is called indirect transrepression. The most important GC anti-inflammatory effects, crucial for patients with inflammatory diseases, take place in this mechanism $[15,16]$. Within the cytoplasm, the active GCGCR complex functions as a monomer and takes part in interactions with many proteins (protein-protein interaction), among them signaling pathways kinases, transcription factors, coactivator proteins, preventing their interactions within the nucleus area. An example is the inhibition of transcription factors, such as activator protein (AP-1) and nuclear factor kappa B (NF $\kappa$ B) and signal transducers and activators of transcription (STAT) [15]. These transcription factors activate the expression of genes responsible for inflammatory processes, antiapoptotic genes and genes regulating cell proliferation. Interaction of protein-protein type with GC-GCR complex wipes out their regulatory effects on gene expression. The GC-GCR complex in the cytoplasm area can also bind proteins with HAT activity (i.e., pCAF, CBP, SRC-1). In this manner the GC inhibits HAT activity directly and recruits HDAC2, which modifies the histone structure into more condensed, limiting the access of transcription factors to DNA ("gene silencing") [15, 17].

Microarray studies of nasal polyps confirmed the wide range of GC genomic actions. Benson et al. [18] reported that fluticasone changed expression of 203 genes, among them 139 of known function, genes associated with inflammation constituting the largest group. It appeared that in nasal polyps inGC not only downregulated proinflammatory genes but also upregulated anti-inflammatory genes. The most highly expressed gene was that producing uteroglobulin. Uteroglobulin is a protein secreted in the lungs by Clara cells, which inhibit leukocyte chemotaxy, A2 phospholipase activity and proinflammatory cytokines activity. Its expression in nasal polyps is considerably decreased (11 times) in comparison with the level of expression observed in healthy mucous membranes [18]. Therefore, a significant increase of uteroglobulin gene expression under the influence of inGC treatment can be a vital element of their activity in nasal polyps.

\section{Nongenomic mechanism of glucocorticoid actions}

For a long time GC effects on gene transcription were considered as the only mechanism of its action. However, the genomic mode of action implies delayed response to GC, as start of specific regulatory protein synthesis occurs at 20-30 min to hours after cell exposition to GC, and only then gradually their activity develops [19]. This was not always compatible with clinical observations. Clinical practice and in vitro and in vivo studies showed some examples of GC therapy effects taking place fast, within minutes or even seconds, in the time excluding protein production de novo [20-25].

Experimental models enable to diversify genomic and nongenomic mechanisms of GC effects. If the activity under study can be observed after transcription (actinomycin D) or translation (cycloheximide) inhibitors administration or in the material of anuclear cells, the nongenomic mode of action is to be implied. The time of biological effects alone does not distinguish precisely both 
Table 1 Molecular mechanisms of glucocorticoids action

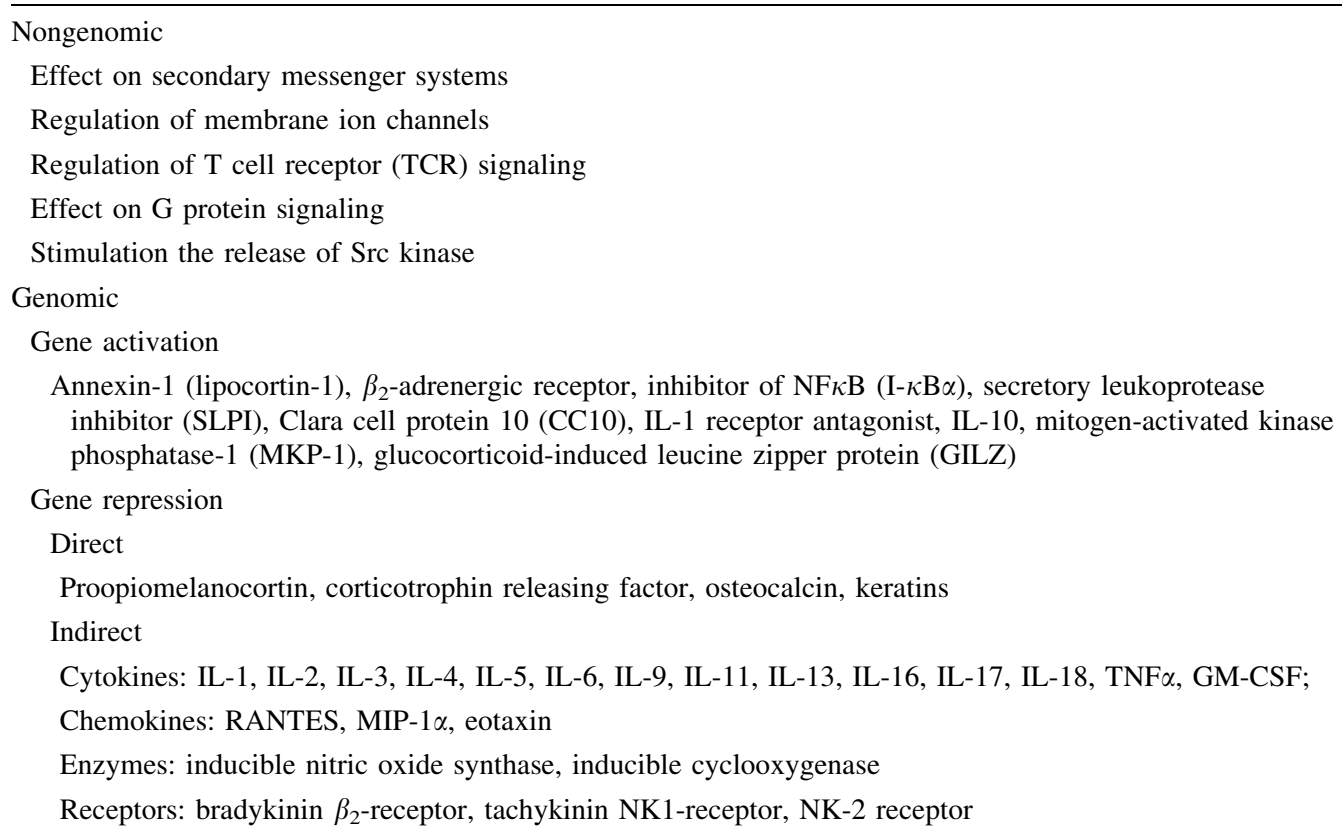

mechanisms because nongenomic ones occurring after longer time cannot be excluded.

GC receptor localized in cell membrane, more rarely in cytoplasm, is responsible for nongenomic GC actions. Nontranscriptive signal can be mediated also without receptor involvement. It was shown that some rapid GC actions are not inhibited by their receptor antagonists. In this mechanism GC activate secondary messengers (calcium ions $\mathrm{Ca}^{2+}$, inositol trisphosphate $\mathrm{IP}_{3}$, diacylglycerol DAG and cyclic nucleotides: cAMP and cGMP). Increase of activity of a secondary messenger induces kinase (or several kinases) activity dependent on it and in consequence changes course of cellular processes. Mitogenactivated protein kinases (MAPK), including mainly protein ERK1/2 and p38 play a substantial role in GC nontranscriptional signals. Other kinases: protein kinase A (PKA) and C (PKC), tyrosine kinase (i.e. Src), lipid kinases (i.e. inositol trisphosphate kinase-PI3K) and signaling pathways related to protein $\mathrm{G}$ and ion channels are also involved [17, 26].

\section{Nongenomic GC effects mediated via membrane glucocorticoid receptors}

Some nongenomic GC effects are mediated via membrane GCR (mGCR). Activated mCCR can stimulate synthesis of secondary messengers and enter into interactions with ion channels protein. There is evidence that rapid GC actions take place through interactions with membrane receptor coupled with G protein (GPCR, G protein coupled receptors). GC, directly or via $\mathrm{mGCR}$, can be bound to Gs protein subunit $\alpha$ activating adenyl cyclase. This contributes to activation of Gs-cAMP-PKA signaling pathway. Membrane GCR is also an important regulator of $\mathrm{T}$ cell receptor (TCR) signaling [26].

\section{Nongenomic GC effects mediated via cytoplasmic glucocorticoid receptors}

In nongenomic action GCR localized in cytoplasm (cGCR) may be involved. Croxtall et al. [23] showed rapid inhibition of arachidonic acid release by dexamethasone which was dependent on the receptor since the reaction did not occur after cell exposition to receptor inhibitor (RU 486). In contrast, its course was not influenced by transcription inhibitor (acitinomycin D). It appeared that binding dexamethasone to GCR caused not only receptor dissociation from Hsp90 chaperones but also a release of Src tyrosine kinases from the complex with Hsp90, which according to the authors inhibited release of arachidonic acid by rapid signaling pathway without any involvement on gene transcription.

\section{Nongenomic GC effects may occur without receptor involvement}

GC-mediated signaling without GCR involvement has been poorly understood. GC, as highly lipophilic compounds, diffuse easily in lipid cell membranes interfering with their 
molecules and influencing their physicochemical properties. This nonspecific GC activity is related mainly to membrane ion (calcium, sodium, potassium, chloride) channels regulation and its result is preserving fluidity and continuity of cell membrane. It was proved that GC megadoses, in a receptor independent mechanism, can inhibit neutrophil degranulation and superoxide anion production within $5 \mathrm{~min}[24,25]$. Next, dexamethasone, reversing the process of intracellular calcium ion accumulation, can decrease secretion of chloride ions through bronchial epithelium [27]. The above-mentioned activities can influence the development of nasal polyps. Berstein et al. [28] believe that intensified transepithelial ion transport, induced by inflammatory condition, is a basic pathologic change in nasal polyps. It makes water relocate into interstitial space, inducing edema and the formation of nasal polyps. According to the authors of this hypothesis, excessive $\mathrm{Na}^{+}$ion absorption and increased $\mathrm{Cl}^{-}$permeability are crucial for this effect [28].

\section{Conditions of nongenomic GC effects activation}

Many aspects of nongenomic GC actions require further studies. The most important question is conditions of nongenomic signaling pathways activation. So far we know that nongenomic mechanisms of GC actions appear especially with higher doses and as a consequence, with higher local ligand concentration in cell. In a randomized, placebo-controlled, double-blind study it was shown that nasal itching was markedly reduced within 10 min after administration of the study drug following either a single high dose of systemic GC, betamethasone $(60 \mathrm{mg})$ or methylprednisolone (400 mg) given intravenously [29]. According to Buttgereit et al. [30] genomic mechanisms can be revealed with the concentration above $10^{-12} \mathrm{M}$, while nongenomic with the concentration of at least $10^{-9} \mathrm{M}$. In contrast, other authors show that preserving fluidity of cell membrane and integration of proteins forming cell membrane (i.e., ion channel proteins, transporting and receptors) require GC concentration above $10^{-4} \mathrm{M}$ [31]. Nongenomic GC effects can also be dependent on GC affinity to its receptor [23]. In this aspect it should be considered that inGC administration ensures high local concentration of the drug (despite low doses applied). The inGC preparations were characterized by considerably higher affinity to its receptor and local potency in comparison to their equivalents administered systemically [32, 33]. Such clinical situation can be favorable for revealing nongenomic mechanisms of inGC actions.

The conditions of all inGC effects are appropriate site of their deposition. One large challenge regarding inGC is insufficient distribution in the nose and paranasal sinuses to mucosal obstruction, especially in cases with nasal polyps. That's why in some cases a good effect with combined treatment using inGC and oral treatment (for short term) is observed [3].

\section{GC effects on the airway vasculature}

The nose has a rich supply of blood vessels. During inflammation, hyperemia, increased blood flow, microvascular permeability, and edema formation occur quite quickly. Some authors claim that it is just large quantities of extracellular fluid that contribute to polyps' development (Bateman's hypothesis) [34]. Moreover, a significant increase of vascular endothelial growth factor (VEGF) expression was shown [35]. This cytokine is a vital angiogenesis stimulator and causes an increase of vessel permeability and dilatation stimulating nitrogen oxide synthesis through endothelium. VEGF also stimulates proteolytic enzymes and expression of receptors important for cell migration and epithelial cells proliferation. The increase in VEGF concentration in nasal lavage from patients with polyposis compared with control subjects is so high (sevenfold higher) that VEGF was acknowledged to be a novel biomarker for chronic rhinosinusitis with hyperplastic sinonasal polyposis. It was also shown that epithelial infection with rhinovirus specifically stimulated VEGF mRNA expression and VEGF release in normal subjects or atopic patients [36].

For the reasons presented above, GC influence on vasculature is an important element of their effectiveness. It was shown that GC exerts rapid, delayed and long-term effects on the airway vasculature [22]. Long-term effects are above all a consequence of GC anti-inflammatory action, and as a result they suppress increased microvascular permeability and oedema formation. GC inhibition of VEGF expression is also significant due to inhibition on the molecular level of oedema formation. Glucocorticoids (as proved by GC given intrabronchially) can also decrease considerably blood flow in mucous membrane vessels. This effect appears quickly, in less than $5 \mathrm{~min}$, which suggests nongenomic type of action [22]. It is thought that GC, in this activity, inhibit extraneuronal uptake of norepinephrine within $\alpha_{1}$-adrenoreceptor in vascular smooth muscle cells [22]. Clinical symptom of this activity is decrease of hyperaemia in the site of inflammation as a result of significant decrease of blood flow in mucous membrane vessels ( $\alpha_{1}$-adrenoreceptor-dependent vasoconstriction of GC).

Local regulation of blood flow and membrane ion channels, can mediate the antisecretory [20] and decongestant effects of inGC in patients with vasomotor rhinitis [37, 38], rhinitis medicamentosa [39] and rhinosinusitis with or without polyps [40]. 


\section{Final remarks}

Intra-nasal GCs are the most important drugs in upper airways diseases. Proving not only slow genomic GC action but also rapid nongenomic mechanisms justifies wider indications for inGC administration in upper airway diseases with mechanism other than classic chronic inflammation such as medicamentosa and vasomotor rhinitis. The presented mechanisms of GC actions indicate that inGCs are effective not only in allergic rhinitis but should also be drugs of first choice in cases coexisting with nonallergic rhinitis (44-87\% cases) [5, 41]. The use of inGC both as monotherapy and adjunctive therapy for acute rhinosinusitis [4] is also supported by the molecular mechanism of GC action. Intra-nasal GCs should be applied regularly for a longer period, but in some cases their occasional use is acceptable (also on an as-needed basis) [5] and this recommendation is based on nongenomic rapid GC effects. InGC should also be drugs of the first choice in the treatment of nasal and sinus polyps because they give a chance of eliminating or decreasing their size. They should be applied as primary treatment in prevention of disease recurrence after surgical treatment. Taking into consideration situations when nongenomic GC effects get revealed, it should be remembered that in some cases the drug doses should be definitely higher than those recommended conventionally. The more that, there are inGC preparations available of high affinity to GCR (which can provide higher chances for disclosing nongenomic actions) and low systemic bioavailability (which provides safety). The current systematic reviews and meta-analyses emphasize their safety as fluticasone propionate and furoate and mometasone furoate systemic bioavailability is $<1 \%$ [42]. Should be glad that the role of inGC is more often recognized as an appropriate and effective treatment option [43].

Conflict of interest statement The authors declare that they have no conflict of interest.

Open Access This article is distributed under the terms of the Creative Commons Attribution Noncommercial License which permits any noncommercial use, distribution, and reproduction in any medium, provided the original author(s) and source are credited.

\section{References}

1. Schalek P, Otruba L, Hahn A (2010) Quality of life in patients with chronic rhinosinusitis: a validation of the Czech version of SNOT-22 questionnaire. Eur Arch Otorhinolaryngol 267:473-475

2. Dudvarski Z, Pendjer I, Djukic V, Janosevic L, Mikic A (2008) The analysis of clinical characteristics of the chronic rhinosinusitis: complicated and uncomplicated form. Eur Arch Otorhinolaryngol 265:923-927

3. Scadding GK, Durham SR, Mirakian R et al (2008) BSACI guidelines for the management of rhinosinusitis and nasal polyposis. Clin Exp Allergy 38(2):260-275

4. Thomas M, Yawn B, Price D et al (2008) EPOS primary care guidelines: European position paper on the primary care diagnosis and management of rhinosinusitis and nasal polyps 2007-a summary. Prim Care Resp J 17(2):79-89

5. Wallace DV, Dykewicz MS, Bernstein DI et al (2008) The diagnosis and management of rhinitis: an updated practice parameter. J Allergy Clin Immunol 122:1-84

6. Bousquet J, Khaltaev N, Cruz AA et al (2008) Allergic rhinitis and its impact on asthma (ARIA) 2008 update. Allergy 63:8-160

7. Shirasaki H, Watanabe K, Kanaizumi E, Konno N, Sato J, Narita S, Himi T (2004) Expression and localization of steroid receptors in human nasal mucosa. Acta Otolaryngol 124(8):958-963

8. Leung DY, Bloom JW (2003) Update on glucocorticoid action and resistance. J Allergy Clin Immunol 111:3-22

9. Pujols L, Mullol J, Torrego A, Picado C (2004) Glucocorticoid receptors in human airways. Allergy 59:1042-1052

10. Pujols L, Mullol J, Picado C (2007) Alpha and beta glucocorticoid receptors: relevance in airway diseases. Curr Allergy Asthma Rep 7:93-99

11. Pujols L, Alobid I, Benitez P, Martinez-Antón A, Roca-Ferrer J, Fokkens WJ, Mullol J, Picado C (2008) Regulation of glucocorticoid receptor in nasal polyps by systemic and intranasal glucocorticoids. Allergy 63:1377-1386

12. Stjärne P, Mösges R, Jorissen M, Passàli D, Bellussi L, Staudinger H, Danzig M (2006) A randomized controlled trial of mometasone furoate nasal spray for the treatment of nasal polyposis. Arch Otolaryngol Head Neck Surg 132(2):179-185

13. Duma D, Jewell CM, Cidlowski JA (2006) Multiple glucocorticoid receptor isoforms and mechanisms of post-translational modification. J Steroid Biochem Mol Biol 102:11-21

14. Bartholome B, Spies CM, Gaber T et al (2004) Membrane glucocorticoid receptors (mGCR) are expressed in normal peripheral blood mononuclear cells and upregulated following in vitro stimulation and in patients with rheumatoid arthritis. FASEB $\mathrm{J}$ 18:70-80

15. Barnes PJ (2006) How corticosteroids control inflammation: quintiles prize lecture 2005. Br J Pharmacol 148:245-254

16. Ito K, Chung KF, Adcock IM (2006) Update on glucocorticoid action and resistance. J Allergy Clin Immunol 117:522-543

17. Stellato C (2004) Post-transcriptional and nongenomic effects of glucocorticoids. Proc Am Thorac Soc 1:255-263

18. Benson M, Carlsson L, Adner M, Jernås M, Rudemo M, Sjögren A, Svensson PA, Uddman R, Cardell LO (2004) Gene profiling reveals increased expression of uteroglobin and other antiinflammatory genes in glucocorticoid-treated nasal polyps. J Allergy Clin Immunol 113:1137-1143

19. George AA, Schiltz RL, Hager GL (2009) Dynamic access of the glucocorticoid receptor to response elements in chromatin. Int $\mathrm{J}$ Biochem Cell Biol 41:214-224

20. Urbach V, Verriere V, Grumbach Y, Bousquet J, Harvey BJ (2006) Rapid anti-secretory effects of glucocorticoids in human airway epithelium. Steroids 71:323-328

21. Verriere V, Hynes D, Faherly S, Devaney J, Bousquet J, Harvey BJ, Urbach V (2005) Rapid effects of dexamethasone on intracellular $\mathrm{pH}$ and $\mathrm{Na}^{+} / \mathrm{H}^{+}$exchanger activity in human bronchial epithelial cells. J Biol Chem 280:35807-35814

22. Horvath G, Vasas S, Wanner A (2007) Inhaled corticosteroids reduce asthma-associated airway hyperperfusion through genomic and nongenomic mechanisms. Pulm Pharmacol Ther 20(2):157-162 
23. Croxtall JD, Choudhury Q, Flower RJ (2000) Glucocorticoids act within minutes to inhibit recruitment of signalling factors to activated EGF receptors through a receptor-dependent, transcription-independent mechanism. Br J Pharmacol 130:289-298

24. Liu L, Wang YX, Zhou J, Long F, Sun HW, Liu Y, Chen YZ, Jiang CL (2005) Rapid non-genomic inhibitory effects of glucocorticoids on human neutrophil degranulation. Inflamm Res 54(1):37-41

25. Long F, Wang YX, Liu L, Zhou J, Cui RY, Jiang CL (2005) Rapid nongenomic inhibitory effects of glucocorticoids on phagocytosis and superoxide anion production by macrophages. Steroids 70:55-61

26. Löwenberg M, Stan C, Holmes DW, Buttgereit F (2008) Novel insights into mechanisms of glucocorticoid action and the development of new glucocorticoid receptor ligands. Steroids 73:1025-1029

27. Urbach V, Walsh DE, Mainprice B, Bousquet J, Harvey BJ (2002) Rapid non-genomic inhibition of ATP-induced $\mathrm{Cl}^{-}$ secretion by dexamethasone in human bronchial epithelium. J Physiol 545:869-878

28. Bernstein JM, Gorfien J, Noble B, Yankaskas JR (1997) Nasal polyposis: immunohistochemistry and bioelectrical findings (a hypothesis for the development of nasal polyps). J Allergy Clin Immunol 99:165-175

29. Tillmann HC, Stuck BA, Feuring M, Rossol-Haseroth K, Tran BM, Lösel R, Schmidt BM, Hörmann K, Wehling M, Schultz A (2004) Delayed genomic and acute nongenomic action of glucocorticosteroids in seasonal allergic rhinitis. Eur J Clin Invest 34:67-73

30. Buttgereit F, Silva JA, Boers M, Burmester GR, Cutolo M, Jacobs J, Kirwan J, Köhler L, Van Riel P, Vischer T, Bijlsma JW (2002) Standardised nomenclature for glucocorticoid dosages and glucocorticoid treatment regimens: current questions and tentative answers in rheumatology. Ann Rheum Dis 61:718-722

31. Haller J, Makara G, Mikics E (2008) The effects of nongenomic glucocorticoid mechanisms on bodily functions and the central neural system. A critical evaluation of findings. Front Neuroendocrinol 29:273-291

32. Salter M, Biggadike K, Matthews JL et al (2007) Pharmacological properties of the enhanced-affinity glucocorticoid fluticasone furoate in vitro and in an in vivo model of respiratory inflammatory disease. Am J Physiol Lung Cell Mol Physiol 293(3):660-667

33. Issar M, Sahasranaman S, Buchwald P, Hochhaus G (2006) Differences in the glucocorticoid to progesterone receptor selectivity of inhaled glucocorticoids. Eur Respir J 27(3): 511-516

34. Newton J, Wong Ah-See K (2008) A review of nasal polyposis. Ther Clin Risk Manag 4(2):507-512

35. Lee HS, Myers A, Kim J (2009) Vascular endothelial growth factor drives autocrine epithelial cell proliferation and survival in chronic rhinosinusitis with nasal polyposis. Am J Respir Crit Care Med 180:1056-1067

36. Psarras S, Volonaki E, Skevaki CL, Xatzipsalti M, Bossios A, Pratsinis H, Tsigkos S, Gourgiotis D, Constantopoulos AG, Papapetropoulos A, Saxoni-Papageorgiou P, Papadopoulos NG (2006) Vascular endothelial growth factor-mediated induction of angiogenesis by human rhinoviruses. J Allergy Clin Immunol 117:291-297

37. Pipkorn U, Berge T (1983) Long-term treatment with budesonide in vasomotor rhinitis. Acta Otolaryngol 95:167-171

38. Webb DR, Meltzer EO, Finn AF Jr, Rickard KA, Pepsin PJ, Westlund R, Cook CK (2002) Intranasal fluticasone propionate is effective for perennial nonallergic rhinitis with or without eosinophilia. Ann Allergy Asthma Immunol 88(4):385-390

39. Hallen H, Enerdal J, Graf P (1997) Fluticasone propionate nasal spray is more effective and has a faster onset of action than placebo in treatment of rhinitis medicamentosa. Clin Exp Allergy 27:552-558

40. Meltzer EO, Caballero F, Fromer LM, Krouse JH, Scadding G (2010) Treatment of congestion in upper respiratory diseases. Int J Gen Med 3:69-91

41. Settipane RA, Charnock DR (2007) Epidemiology of rhinitis: allergic and nonallergic. Clin Allergy Immunol 19:23-34

42. Kalish LH, Arendts G, Sack R, Craig JC (2009) Topical steroids in chronic rhinosinusitis without polyps: a systematic review and meta-analysis. Otolaryngol Head Neck Surg 141(6):674-683

43. Cingi C, Songu M (2009) Nasal steroid perspective: knowledge and attitudes. Eur Arch Otorhinolaryngol. doi:10.1007/s00405009-1159-5 